\title{
Avaliação do equilíbrio, agilidade e presença de tontura em pacientes com migrânea com e sem aura
}

\author{
Carvalho GF, Gonçalves MC, Florêncio LL, Dach F, Ferreira KS, Chaves TC, Speciali JG, Bevilaqua-Grossi D \\ Faculdade de Medicina de Ribeirão preto, Universidade de São Paulo - USP
}

\begin{abstract}
Carvalho GF, Gonçalves MC, Florêncio LL, Dach F, Ferreira KS, Chaves TC, Speciali JG, Bevilaqua-Grossi D. Avaliação do equilíbrio, agilidade e presença de tontura em pacientes com migrânea com e sem aura
\end{abstract}

Headache Medicine. 2012;3(4):200-3

\section{INTRODUÇÃO}

A migrânea pode estar associada ao acidente vascular cerebral e a complicações isquêmicas devido à depressão da atividade elétrica e consequente diminuição do fluxo sanguíneo cerebral. ${ }^{(1,2)}$ A substância branca cerebral é afetada, especialmente nas regiões do cerebelo, tronco encefálico e o ouvido interno, podendo favorecer o aparecimento de sinais e sintomas como vertigem, desequilíbrio, aumento da oscilação postural, incoordenação dos movimentos, dismetria e/ou disfagia, além de prejuízos na força e ritmo da contração muscular. ${ }^{(3)}$ Segundo Etminan (2005) essas alterações são maiores quando há a presença de aura. ${ }^{(1)}$

\section{OBJETIVO}

Avaliar a oscilação do centro de pressão (COP), a agilidade, o relato e impacto da tontura em pacientes com migrânea com aura (MA) e sem aura $(M)$ e indivíduos controle (GC).

\section{MÉTODOS}

Os pacientes, todos do gênero feminino e idade entre 18 e 55 anos, foram triados do ambulatório de cefaleia de um hospital terciário e diagnosticadas com migrânea com aura ou sem aura por neurologistas especialistas em cefaleia de acordo com a ICHD-II (2004). Foram excluídos pacientes obesos, com relato de vestibulopatias, doenças sistêmicas, outros tipos de cefaleia ou em uso de medicamentos que afetem o equilíbrio. $\bigcirc$ grupo controle foi composto de funcionários e acompanhantes, pareados pela idade.

Foram avaliadas 92 voluntárias, sendo 31 do grupo M, 31 do grupo MA e 30 do GC. A amostra foi homogênea, exceto pelo índice de massa corporal (IMC), maior no grupo MA em relação ao GC (Tabela 1).

\begin{tabular}{|c|c|c|c|c|c|c|}
\hline & \multicolumn{2}{|c|}{$\begin{array}{c}G C \\
(n=30)\end{array}$} & \multicolumn{2}{|c|}{$\begin{array}{c}M \\
(n=31)\end{array}$} & \multicolumn{2}{|c|}{$\begin{array}{c}\mathrm{MA} \\
(\mathrm{n}=31)\end{array}$} \\
\hline & Média & $\mathrm{DP}$ & Média & DP & Média & $\mathrm{DP}$ \\
\hline Idade (anos) & 33 & 9 & 38 & 10 & 37 & 8 \\
\hline IMC (kg/cm2) & 23 & 3 & 25 & 5 & $26^{*}$ & 3 \\
\hline $\begin{array}{l}\text { Tempo de doença } \\
\text { (anos) }\end{array}$ & - & 18 & 13 & 18 & 12 & \\
\hline $\begin{array}{l}\text { Frequência } \\
\text { mensal de crises }\end{array}$ & - & 7 & 3 & 7 & 3 & \\
\hline $\begin{array}{l}\text { Intensidade da dor } \\
\text { (EVA) }\end{array}$ & - & 8 & 1 & 8 & 2 & \\
\hline
\end{tabular}

O COP foi mensurado através da estabilometria com uma plataforma de força (AMTI - OR6-7-1000) em apoio bipodal e unipodal, com olhos abertos e fechados durante trinta segundos. A coleta foi aleatorizada por sorteio.

A agilidade foi avaliada pelo teste Timed Up and Go (TUG), no qual o paciente é orientado a se levantar de uma cadeira, andar três metros e retornar a sentar em um menor tempo possível.

A tontura foi avaliada pelo questionário Dizziness Handicap Inventory (DHI), que considera os aspectos psicológicos, físicos e funcionais relacionados a este sintoma.

Para análise entre os grupos, foi utilizado um modelo de regressão linear com efeitos mistos para a plataforma 
de força e teste TUG e um modelo de regressão logística para o DHI, com nível de significância de $\alpha<0,05$.

estudo foi aprovado pelo comitê de ética local (processo $\left.n^{\circ} 4126 / 2010\right)$.

\section{RESULTADOS}

Os resultados revelaram maior oscilação do COP no grupo MA em relação ao grupo $\mathrm{Me} \mathrm{GC}$ em apoio bipodal com olhos abertos e fechados $(p<0,02)$. Pacientes com MA apresentaram maior deslocamento do COP em relação ao grupo $M$ nas situações unipodal com olhos abertos $(p<0,02)$ (Figura 1). Os pacientes com migrânea não conseguiram executar a coleta em apoio unipodal com os olhos fechados, sendo este dado dispensado da análise. Os dados de controle dos pacientes, inclusive o IMC, não

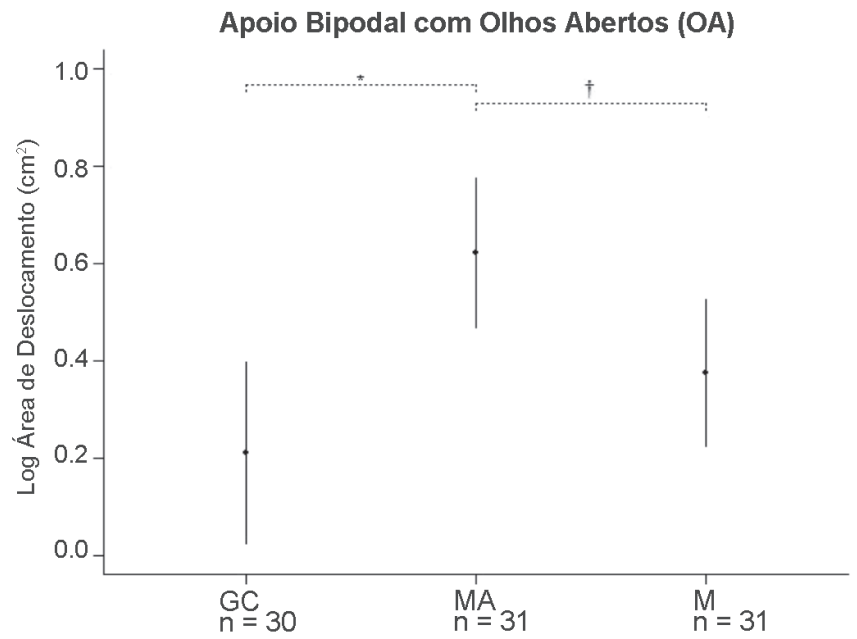

Apoio Unipodal Direito com Olhos Abertos (DOA)

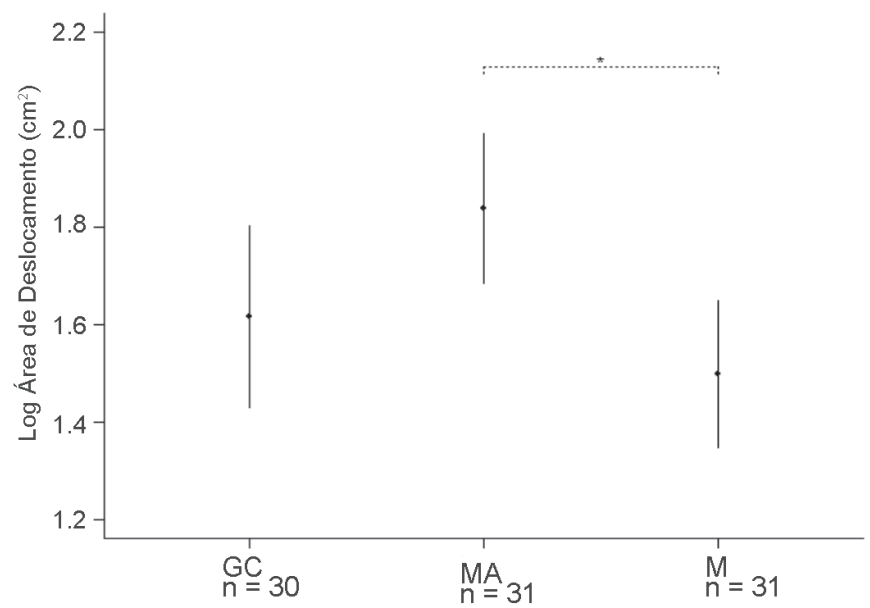

exerceram influência nos dados da plataforma de força, teste TUG e relato de tontura.

Não houve diferença no tempo de realização do TUG (segundos) entre os grupos MA (8,5 segundos) e $M(8,2$ segundos), porém ambos os grupos foram diferentes do $G C(6,5$ segundos; $p<0,01)$ (Figura 2). Além da presença da migrânea, o aumento da idade se mostrou relacionado com a diminuição da mobilidade.

Sintomas de tontura foram verificados em $80 \%$ do grupo $M A$ e $65 \%$ do grupo $M$, apresentando um risco de 58 vezes no grupo MA e de 25 vezes no grupo $M$ para o desenvolvimento deste sintoma em relação a indivíduos sem a patologia. A tontura exerceu influência significante nas atividades de vida diária destes pacientes, com impacto moderado segundo os escores do questionário $(p<0,001)$ (Tabela 2).

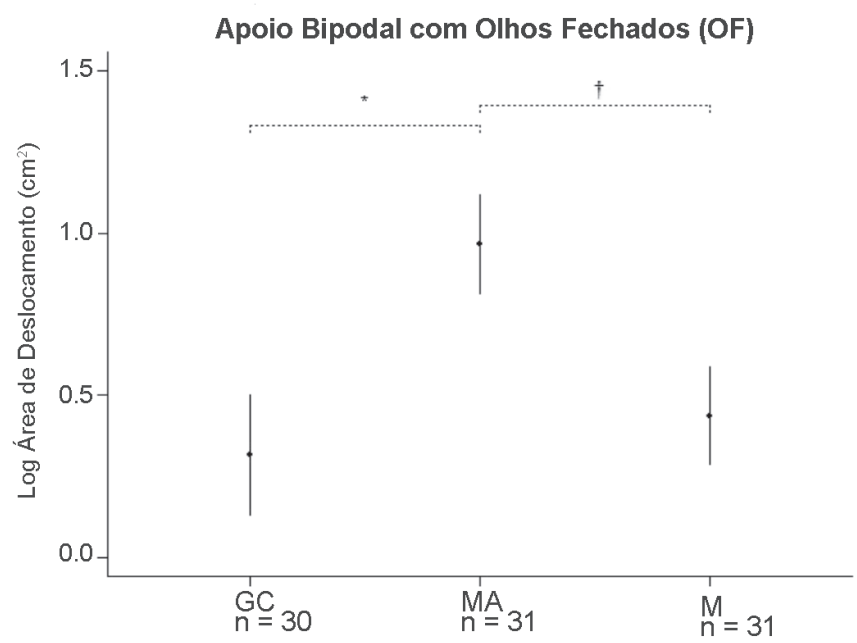

Apoio Unipodal Esquerdo com Olhos Abertos (EOA)

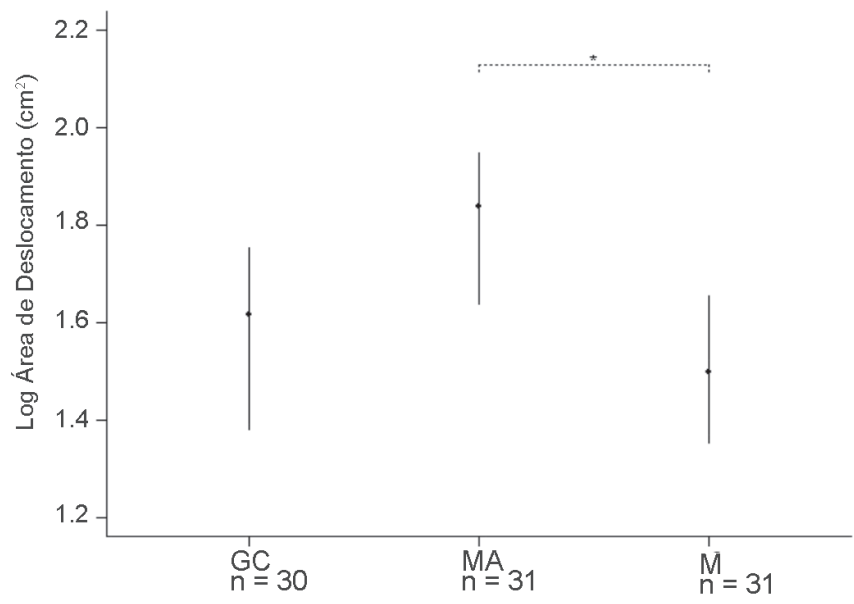

Figura 1 - Log. da média e intervalo de confiança das áreas de oscilação corporal $\left(\mathrm{cm}^{2}\right)$ em apoio bipodal com olhos abertos (OA), olhos fechados (OF), Apoio unipodal direito (DOA) e esquerdo (EOA) com olhos abertos do Grupo Controle (GC), Grupo Migrânea sem Aura (M) e Grupo Migrânea com Aura (MA). ${ }^{* *}$ Transformação da variável em Logaritmo. ${ }^{*} p=0,001 ;{ }^{+} p=0,02$. 


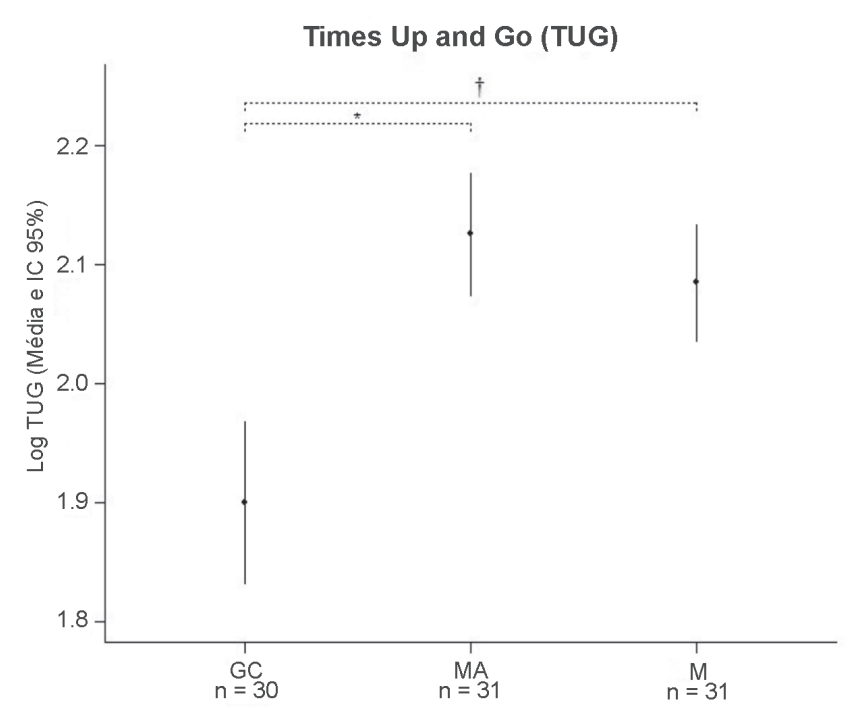

Figura 2 - Log. da média e intervalo de confiança das áreas do tempo de execução do teste Timed Up and Go (TUG).

${ }^{*} p<0,001 ;{ }^{\dagger} p<0,01$.

Tabela 2 - Relato de Tontura (\%), Odds Ratio para o desenvolvimento da tontura e escores atingidos no $\mathrm{DHI}$

\begin{tabular}{lccc}
\hline & $\begin{array}{c}\text { GC } \\
(n=30)\end{array}$ & $\begin{array}{c}\text { M } \\
(n=31)\end{array}$ & $\begin{array}{c}\text { MA } \\
(n=31)\end{array}$ \\
\hline Relato de Tontura (\%) & $6,5 \%{ }^{*}$ & $65 \% * *$ & $80 \%$ \\
Odds Ratio [Estimativa (IC)] & - & $25(5-127)$ & $58(10-315)$ \\
DHI [Média (DP)] ${ }^{1}$ & $0,67(3,6)^{\dagger}$ & $34,4(28,6)$ & $36,1(23,6)$ \\
\hline
\end{tabular}

GC: Grupo Controle; M: Grupo Migrânea sem Aura; MA: Grupo Migrânea com Aura; IC: Intervalo de Confiança; DP: Desvio Padrão. ${ }^{1}$ Escore -100.

${ }^{*} G C$ versus $M$ e MA. $p<0,0001 .{ }^{* *} M$ versus $M A . p<0,001 .{ }^{\dagger} G C$ versus $M e$ MA. $p<0,001$.

\section{DISCUSSÃO}

Nossos resultados mostraram que houve maior oscilação em pacientes com migrânea em nas situações OA e OF, contudo, houve uma diferença maior entre os grupos quando o teste foi realizado com os olhos fechados. Outros estudos iá sugeriram uma tendência de deslocamento maior em migranosos e piores resultados nos testes com olhos abertos ${ }^{(4)}$ ou com olhos fechados. ${ }^{(3)}$

É importante salientar que os indivíduos com migrânea com aura apresentaram prejuízo quanto à oscilação postural nas posições de apoio bipodal e unipodal com olhos abertos, todavia, foram capazes de realizar a função. Já com apoio unipodal e olhos fechados, especialmente com o membro não dominante, ambos os grupos com migrânea apresentaram uma limitação funcional para esta tarefa. Este dado sugere maior risco de quedas em situações que exijam tal função, como em atividades diárias onde haja diminuição da luminosidade, por exemplo.

Nossos resultados também apontaram para a influência da idade e da migrânea sobre os resultados do teste TUG. É possível inferir que idosos com história de migrânea apresentem um aumento particular do risco de quedas quando comparado a idosos sem migrânea.

Além da idade, a cronicidade parece ser um fator agravante para o desequilíbrio. ${ }^{(5)}$ Em pacientes com migrânea crônica foram detectadas disfunções subclínicas nos sistemas vestibular e cerebelar, além de alterações da motricidade ocular, sugerindo que a maior oscilação tenha causa central. ${ }^{(5,6)}$ Esses achados são coerentes com estudos de imagem que relacionam o aumento da frequência de crises com a maior incidência de lesões corticais, encontradas especialmente no cerebelo de migranosos com aura. ${ }^{(1,2)}$ É possível que em uma amostra composta de pacientes com migrânea crônica fosse possível verificar um maior espectro de diferença em relação ao grupo controle.

Apesar de estar bem estabelecida a associação entre as patologias vestibulares e relato de vertigem/tontura em pacientes com migrânea, ${ }^{(7)}$ pouco se sabe sobre 0 impacto dessas condições nesse grupo de pacientes.

Os dados revelaram que houve um impacto moderado da tontura nos aspectos físicos, funcionais e psicológicos nos pacientes com migrânea, independente da presença de aura. Escores semelhantes do DHI foram encontrados em outros estudos. A melhora dos escores do $\mathrm{DHI}$ com a reabilitação vestibular ${ }^{(8)}$ sugere que estes pacientes precisem ser orientados quanto ao risco de quedas em atividades funcionais, especialmente durante a crise.

\section{CONCLUSÕES}

Nossos dados demonstraram que pacientes com migrânea (com e sem aura) apresentam alterações no equilíbrio estático, na agilidade e mobilidade, além da alta prevalência de tontura, com impacto em vários aspectos da vida diária. A aura parece influenciar de forma significativa a estabilidade postural destes pacientes.

\section{REFERÊNCIAS}

1. Etminan M, Takkouche B, Isorna FC, Samii A. Risk of ischaemic stroke in people with migraine: systematic review and metaanalysis of observational studies. BMJ. 2005;330:63. 
2. Swartz RH, Kern RZ. Migraine Is Associated With Magnetic Resonance Imaging White Matter Abnormalities A Metaanalysis. Arch Neurol. 2004;61:1366-8.

3. Ishizaki K, Mori N, Takeshima T, Fukuhara Y, ljiri T. Static stabilometry in patients with migraine and tension-type headache during a headache-free period. Psychiatry Clin Neurosci. 2002; 56(1):85-90.

4. Rossi C. et al. Balance disorders in headache patients: evaluation by computerized static stabilometry. Acta Neurol Scand. 2005; $111(6): 407-13$
5. So CW, Bent LR. Increased vestibular contribution to posture control in individuals with chronic headache. J Vestib Res. 2009; 19(1-2)49-58.

6. Harno H, et al. Subclinical vestibulocerebellar dysfunction in migraine with and without aura. Neurology. 2003;61(12): 1748-52.

7. Parker, W. Migraine and the vestibular system in adults. Am J Otol. 1991;12(1):25-34.

8. Wrisley DM, Whitney SL, Furman JM. Vestibular rehabilitation outcomes in patients with a history of migraine. Otol Neurotol. 2002; 23(4):483-7. 